\title{
An Improved Data Scheduling Mechanism of Streaming Media in Mobile Network
}

\author{
Chengyu Zheng \\ China Telecom Sichua Branch, \\ Chengdu 610041, China. \\ zheng.chengyu@189.cn
}

\author{
Xiaotao Huang* \\ College of Computer Science and \\ Technology Huazhong University \\ of Science and Technology, \\ Wuhan 430074, China. \\ huangxt@mail.hust.edu.cn \\ * the corresponding author
}

\author{
Xiaochen Cheng \\ College of Computer Science and \\ Technology Huazhong University \\ of Science and Technology, \\ Wuhan 430074, China. \\ 1325894618@qq.com
}

\begin{abstract}
The gradual evolution of mobile communication network into $4 \mathrm{G}$ is increasingly making the streaming media become a new attractive business. However, the service quality of mobile streaming media is urgent to be improved for better service. The relatively fair data scheduling mechanism, node data scheduling within cell and node data scheduling when switching cells are presented in terms of mobile feature of mobile streaming media nodes. The wireless access point selection for node data scheduling within cell is further researched, and a wireless access point selection mechanism which is suitable for mobile streaming media is provided. The theoretic analysis verifies the fair principle of user service quality for mobile streaming media, and the appropriate data scheduling mechanism improves the reliability of data transmission, which entirely boosts the service ability of system. The simulation test shows that the wireless access point selection mechanism balances the loads of wireless access points to some extent and improves the node number of entire system.
\end{abstract}

\section{Keyword}

mobile network, streaming media, data scheduling mechanism, node selection mechanism

\section{INTRODUCTION}

The development of third generation mobile communication network technology, enforcement of mobile terminal functions and abundance of mobile business content are increasingly facilitating people's daily life. As the mobile communication network evolves into 4G gradually, the more amusing mobile streaming media businesses such as mobile TV and VOD by mixing the sound, text and image will certainly become a new service. Thus, a new attractive business, namely the mobile streaming media business, emerges under the circumstance ${ }^{[1-3]}$.

The application of traditional streaming media technology into the mobile one will generally encounter two problems ${ }^{[4-7]}$. One is the instability of wireless network, limited bandwidth, largely fluctuated data transmission speed and so on, which affects the service quality greatly. Another is that the storage capacity and processing capability of mobile terminals are much lower in comparison with PC. The location and movement speed of mobile terminals vary at any time, which results into more difficult in data scheduling and bandwidth allocation of traditional streaming media. The features above bring some problems, for example, how the limited bandwidth satisfies the more service requests, how the streaming media are played smoothly at the cell boundary, and how the data are scheduled reasonably in case of instable wireless network and limited terminal capacity.

This paper aims at solving some problems of mobile streaming media application to improve the service quality of mobile streaming media. Based on the existing streaming media technology, a system model which applies to mobile streaming media is designed in consideration of the application circumstance of mobile streaming media. The bandwidth allocation and data scheduling of given model are discussed. The data scheduling mechanism, node data scheduling within cell and node data scheduling when switching cells are presented in terms of mobile feature of mobile streaming media nodes. The wireless access point (AP) selection for node data scheduling within cell is further researched, and a wireless AP selection mechanism that is suitable for mobile streaming media is provided.

\section{Problems AND MODElS}

The models of streaming media are generally classified into centralized model, pure peer-to-peer model and mixed peer-topeer model[8]. The system model in this paper is mixed peer-topeer model according to the architecture in literature [9]. The model is divided into two layers, as shown in Fig. 1. One layer consists of streaming media server and proxy server. Another is the data transmission between cell nodes. This system has the following components and functions:

(1) Streaming media server S0: it basically receives the requests from proxy servers and buffers the information about proxy servers into a simple index server. It balances the loads of various proxy servers by appropriate scheduling and brings better cooperation between proxy servers. In addition, coding video is finished by streaming media server.

(2) Streaming media proxy server S1-S6: they are on the verge of network in Fig. 1. They can store and manage the video 
programs, serve as the index servers for cell nodes for information inquiry, and provide video stream for mobile media nodes.

(3) Gateway node: access points must connect to the backbone network by gateway, and it can connect to various data networks by gateway.

(4) Access point: it is the intermediate node of wireless communication. The wireless terminals send and receive data through AP. Thus, the mobile streaming media node must associate with some AP to communicate with other mobile streaming media modes or visit the external network.

(5) Authentication and authorization server: when the mobile node moves from current AP cell to new AP cell, this serve will disconnect the current AP immediately and begin to authenticate and reconnect the new AP.

(6) Mobile streaming media nodes: they mainly decode and play the video, communicate with other terminals (other mobile streaming media nodes and corresponding proxy servers), and send video data.

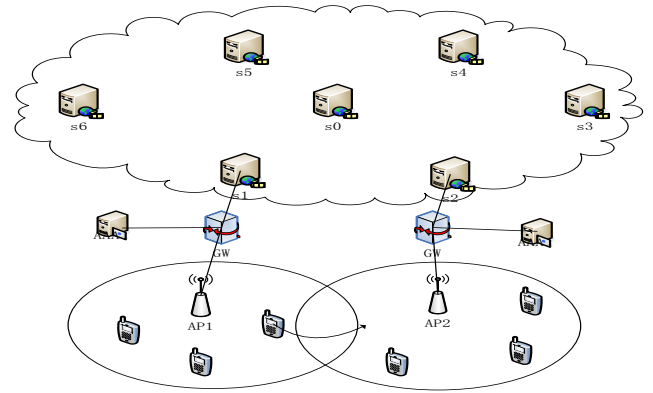

Fig. 1 Model of mobile streaming media system

This system uses the cell unit to send data, and the advantages of introducing the proxy servers are shown as follows. Firstly, the proxy servers can provide stable and continuous stream service for better quality in case of instable wireless network, mobile media nodes and uncertainty of closing or starting the servers. Secondly, the proxy server can function as index server in cell and takes the task to search stream media resources. Thus, the proxy server can control the information redundancy in the whole mobile network and avoid the decreasing of transmission quality due to the network congestion. The layered multi-description coding is used for mobile streaming media[10], and literature [11] provides the strategy of bandwidth allocation. In this paper, the allocation is classified into data scheduling within cell and data scheduling when switching cells[12-14] based on the mobile path of mobile node. The scheduling is studied in the view of node layer and proxy server layer.

Question 1: mobile streaming media data scheduling within cell

The data scheduling for cable streaming media can buffer much video content in advance due to the abundant bandwidth and better-performance terminals. However, it is difficult to buffer much video content in advance in mobile streaming media because of the instable wireless network, limited bandwidth, and limited storage capacity and performance of mobile nodes. Too much bandwidth cannot be provided to a single mobile node to transfer much video content in unit time, and buffering much video may affect the performance of mobile node. If much video is buffered in advance, the instable mobile node may result in the close of service, which wastes too much buffered video. In addition, many video clips are discarded due to the limited mobile node storage capacity because the streaming files must be played continuously, which heavily wastes the bandwidth. So, it is necessary to present a reasonable data scheduling mechanism.
Question 2: data scheduling when switching cells

The mobile streaming media node is generally in motion, and the wireless network is geographically regular hexagon cell. In each cell, the wireless AP sends signal covering the entire cell to provide service for mobile media nodes. For example, the AP will be switched if the train or bus crosses from one cell to another cell when we watch live sporting event on the mobile phone. During this period, the streaming media will disconnect with original wireless AP, pause streaming media service, then reconnect with new wireless AP, and start the streaming media service. The period of service pause during wireless switch will affect the streaming media service quality to large extent. Thus, it is important to minimize the negative influence and ensure the service quality.

Question 3: data scheduling of streaming media proxy server

The mobile streaming media node may request any video at any time, so the proxy server and other nodes in the cell where the node is located should find the video requested and send it to the node as soon as possible to reduce the waiting time. If the video requested is not found in this cell, the video should be searched in other cells and then be sent to this cell. In addition, some video should be sent to the proxy server of next possibly reaching cell because the mobile node moves uncertainly. The proxy server is easily buffered much unnecessary video due to the mobile node motion and service pause at any time.

\section{MODEL AND DEFINITIONS}

Assume a mobile streaming media node $\mathrm{q}_{\mathrm{i}}$ located aside point A in the cell covered by wireless AP ap1 moves at an even speed of $\mathrm{V}$ along line $\mathrm{AE}$ from ap1-covered cell to ap2-covered cell, as shown in Fig. 2. The moving path can be divided into three sections: line $\mathrm{AB}$ is only at ap1-covered cell, line $\mathrm{BD}$ at overlap zone of ap1 and ap2, and line BE at ap2-covered cell.

The system in this paper presents the solution in Fig. 3. The whole video is divided into terms of unit chip of playing time $\mathrm{T}$, and then is coded by layered multi-description method.

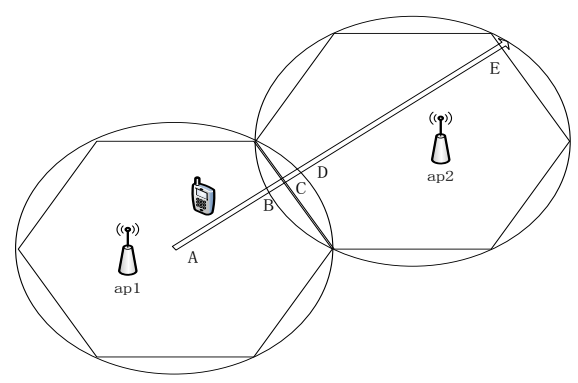

Fig. 2 Moving path of node

Assume that the mobile node $\mathrm{q}_{\mathrm{i}}$ at point $\mathrm{A}$ requests the first chip of video $R 1$, and the $S^{\text {th }}$ chip of video R1 is watched when $q_{i}$ arrives at point $\mathrm{B}$. For continuous playing in various periods, the video chips should satisfy: 1) the first to $S^{\text {th }}$ chips are needed during the period of

$$
\begin{array}{r}
T_{a b}=\frac{|A B|}{V} \\
\text { 2) the }(\mathrm{S}+1)^{\text {th }} \text { to } \mathrm{M}^{\text {th }} \text { chips are needed during } \\
T_{a b}=\frac{|B A|}{V} \\
\text { and }(\mathrm{M}+1)^{\text {th }} \text { to }^{\text {th }} \text { chips are needed during } \\
T_{c d}=\frac{|C D|}{V}
\end{array}
$$$$
\text { . Thus, } T_{b c}=T_{c d}, 2 \mathrm{M}=\mathrm{S}+1+\mathrm{N} \text {. }
$$ 


\begin{tabular}{|l|l|l|l|l|l|l|l|l|l|l|l|l|l|}
\hline 1 & 2 & 3 & $\ldots$ & $\mathrm{S}$ & $\mathrm{S}+1$ & $\ldots$ & $\mathrm{M}$ & $\ldots$ & $\mathrm{N}$ & $\ldots$ & $\ldots$ & $\ldots$ & $\mathrm{T}$ \\
\hline
\end{tabular}

Fig. 3 Scheduling series of video clips

Definition 1: estimated leaving period tpl(i) of mobile node: the interval from current position to departing from cell when the $i^{\text {th }}$ node moves at some given speed.

Definition 2: switching-leaving period $\mathrm{tl}(\mathrm{i})$ of mobile node: the interval from detecting the cell by the $i^{\text {th }}$ node to leaving the cell.

Definition 3: transmission time tb(i) of base layer: time needed by the $\mathrm{i}^{\text {th }}$ node to download the base layer data of each video clip with download bandwidth.

Definition 4: enhancement layer sub-description transmission time te(i) : time needed by the $i^{\text {th }}$ node to download the enhancement layer sub-description data of each video clip with download bandwidth.

Definition 5: node video number $\mathrm{R}\left(q_{i}\right)$ : the number of total video resources owned by mobile node.

Definition 6: scheduling node available for base layer: the $i^{\text {th }}$ node contains base layer content of some video chip and the residual upload bandwidth of the $\mathrm{i}^{\text {th }}$ node can transmit the base layer data of this chip, with $\operatorname{tpl}(\mathrm{i}) \geq \mathrm{tb}(\mathrm{i})$.

Definition 7: scheduling node available for sub-description: the $\mathrm{i}^{\text {th }}$ node contains sub-description content of some video chip and the residual upload bandwidth of the $\mathrm{i}^{\text {th }}$ node can transmit the subdescription data of this chip, with $\operatorname{tpl}(\mathrm{i}) \geq t e(i)$.

\section{NODE SCHEDULING MECHANISM AND ANALYSIS}

\subsection{Node Scheduling Mechanism Within Cell}

The data scheduling mechanism for motion line $A B$ is discussed in this section, which is suitable for the mobile node moving in only one cell which is covered by one wireless AP. The line DE is similar with $\mathrm{AB}$, so the scheduling mechanism of $\mathrm{DE}$ will be not discussed. The following will deal with the data scheduling of the first chip at point A.

The algorithm focuses mainly on translating the data scheduling of unit video chip into the data scheduling of base layer and various sub-descriptions. Find the node set available for base layer and various sub-descriptions, and then sort then in ascending order in terms of tpl(i). The node with smaller residual upload bandwidth will be selected for data transmission if the minimal tpl(i) $>\operatorname{tl}(\mathrm{i})$, otherwise the node with minimal tpl(i) will be selected for data transmission. The algorithm is described as follows:

(1) Detect the current download bandwidth $\mathrm{BW}\left[\mathrm{q}_{\mathrm{i}}\right]$ available for mobile node.

(2) Translate the download bandwidth of mobile node into corresponding base layer and $\mathrm{n}$ sub-description.

(3) Data scheduling node set $\mathrm{A}_{\mathrm{q}}$ available for base layer.

(4) The proxy server provides data scheduling of base layer if $\mathrm{A}_{\mathrm{q}}$ is null.

(5) Else sort $\mathrm{A}_{\mathrm{q}}$ nodes in ascending order in terms of $\operatorname{tpl}\left(q_{i}\right)-$ $t l\left(q_{i}\right)$.

(6) Select nodes with min $\left(\operatorname{tpl}\left(q_{i}\right)-t l\left(q_{i}\right)\right)$ for data scheduling if $\min \left(t p l\left(q_{i}\right)-t l\left(q_{i}\right)\right)<0 \& \& \operatorname{count}\left(\min \left(t p l\left(q_{i}\right)-t l\left(q_{i}\right)\right)\right)=1$.

(7) Select nodes with min $\left(\operatorname{tpl}\left(q_{i}\right)-t l\left(q_{i}\right)\right)<0 \& \& \min \left(R\left(q_{i}\right)\right)$ for data scheduling else if $\min \left(\operatorname{tpl}\left(q_{i}\right)-t l\left(q_{i}\right)\right)<0 \& \& \operatorname{count}\left(\min \left(\operatorname{tpl}\left(q_{i}\right)-t l\left(q_{i}\right)\right)\right)>1$.

(8) Else sort $\mathrm{A}_{\mathrm{q}}$ nodes in ascending order in terms of BW $\left[q_{i}\right]$.

(9) Select nodes with min (BW $\left[q_{i}\right]$ ) for data scheduling if $\operatorname{count}\left(\min \left(\mathrm{BW}\left[q_{i}\right]\right)\right)=1$.
(10) Else Select nodes of min $\left(B W\left[q_{i}\right]\right) \& \& \min (R(i))$ for data scheduling.

(11) The calculation steps of data scheduling of sub-description are identical with that of base layer.

\subsection{Node Scheduling Mechanism Between Cells}

\subsubsection{Data Scheduling Mechanism for Overlap Zone Between Two Cells}

The data scheduling mechanism for line BD is discussed in this section. In other words, the data scheduling mechanism is analyzed when mobile node moves in the overlap zone between two cells covered by wireless APs

Definition 8: switching-detected point: when mobile node arrives at the cell boundary, the occurrence of switching will be detected.

Definition 9: disconnection point for wireless AP: when mobile node arrives at this point, it will disconnect the wireless AP of original cell.

Definition 10: connection point for wireless AP: when mobile node arrives at this point, it will connect the wireless AP of new cell.

Definition 11: switching time $T_{h}$ : the time of mobile node switched from disconnection point to connection point.

According to the definitions above, the zone $\mathrm{BD}$ is the switching zone. When mobile node $q_{i}$ arrives at point $\mathrm{B}$, switching will be detected at point $B$. The ap1-covered cell boundary cuts the moving path of node $q_{i}$ at point $\mathrm{C}$ called disconnection point for wireless AP. When mobile node $q_{i}$ arrives at point $\mathrm{D}$ at ap2-covered cell, it will connect with ap2, with point $\mathrm{D}$ called connection point for wireless AP. Thus,

$$
T_{h}=\frac{|C D|}{V} \text {. }
$$

The mobile node cannot get new video clips during switching time $\boldsymbol{T}_{\boldsymbol{h}}$ due to the service pause of streaming media. Thus, the scheduling mechanism presented need ensure that the video should be played continuously during this period. To achieve the goal, the algorithm in this paper buffers some video chips in advance needed for switching time $\boldsymbol{T}_{\boldsymbol{h}}$. For the period from switching-detected point to disconnection point, not only the clips for this period but also the clips for switching time $\boldsymbol{T}_{\boldsymbol{h}}$ are buffered. In addition, the algorithm should control the buffered video number to avoid the waste. The algorithm procedures are described as follows:

(1) Detect the current download bandwidth $\mathrm{BW}\left[\mathrm{q}_{\mathrm{i}}\right]$ available for mobile node.

(2) Determine the base layer and sub-descriptions translated by download bandwidth available.

(3) Determine the switching-detected point B and assume that the $S^{\text {th }}$ clip is played at present at point $B$.

(4) The data scheduling for $(\mathrm{S}+1)^{\text {th }}$ clip and $(\mathrm{M}+1)^{\text {th }}$ clip is conducted by the algorithm in section 4.3.1.

(5) The data scheduling for $(\mathrm{S}+1)^{\text {th }}$ clip includes the base layer and sub-descriptions:

$$
\frac{B W\left[q_{i}\right]-2 * a}{b}
$$

(6) The data scheduling for $(\mathrm{M}+1)^{\text {th }}$ clip only includes the base layer. 


\subsubsection{Data Scheduling Mechanism for Overlap Zone} Among Multiple Cells

When the mobile node moves from one cell to overlap zone between two cells and then to overlap zone among multiple cells, it may select the wireless AP if it moves long time in the overlap zone among multiple cells. The appropriate wireless AP should be selected at first, and then the data scheduling will be discussed.

Definition 12: signal intensity S(i) of wireless AP: the signal intensity of the $\mathrm{i}^{\text {th }}$ wireless AP: AP(i).

Definition 13: video resource number R(i): the number of video clips needed by proxy servers and other nodes (including switching mobile nodes) in the cell of the $i^{\text {th }}$ wireless AP: AP(i).

Definition 14: bandwidth available APAW(i): the size of bandwidth available for the $i^{\text {th }}$ wireless AP: AP(i).

Definition 15: performance value of wireless AP: $P_{i}=$ $\mathrm{S}(\mathrm{i})[\alpha * \operatorname{APAW}(\mathrm{i})+(1-\alpha) * \mathrm{R}(\mathrm{i})]$, weigh the service ability of $\mathrm{AP}$ and corresponding proxy server, and the coefficient $a$ is determined by specific network environment and practical experiences.

a) AP selection mechanism

The mobile streaming media node need select the wireless AP when it switches the cell. Some factors, like signal intensity of wireless AP, whether new cell has the video the node needs, and bandwidth of new cell, at this moment will affect the service quality of mobile streaming media after the selection of wireless AP. A good selection mechanism of AP must ensure the continuous service quality, system service ability, and load balance. The algorithm procedures are described as follows:

(1) Determine the data scheduling size (the video clip series) for the mobile node in the switching zone.

(2) The mobile node detects the signal intensity $\mathrm{S}(i)$ of each wireless AP and the bandwidth $\operatorname{APAW}(i)$ available for the cell where each wireless AP is located.

(3) Determine statistically the video resource number $\mathrm{R}(i)$ available for the cell where each wireless AP is located based on the video clip series from step 1 .

(4) Calculate $\mathrm{P}_{\mathrm{i}}=\mathrm{S}(\mathrm{i})[\alpha * \operatorname{APAW}(\mathrm{i})+(1-\alpha) * \mathrm{R}(\mathrm{i})]$ by $\mathrm{S}(i), \operatorname{APAW}(i)$ and $\mathrm{R}(i)$, sort them in ascending order, and connect the wireless AP with maximum $\mathrm{P}(i)$.

b) Data scheduling scheme

The change in $\mathbf{S}(\boldsymbol{i}), \mathbf{A P A W}(\mathbf{i})$ and $\mathbf{R}(\mathbf{i})$ will affect the data scheduling mechanism of mobile node. The possible data scheduling schemes are provided in this section according to the change of $\mathbf{S}(\boldsymbol{i})$, APAW $(\mathbf{i})$ and $\mathbf{R}(\mathbf{i})$.

(1) $\mathrm{S}(i)=0 \quad 1 \leq i \leq N$

In this case, the mobile node cannot receive any AP signal, and also cannot provide any streaming media service. The mobile node is at the zone with no signal, so enough video clips should be buffered before switching to satisfy the playing need in the switching zone.

(2) $\exists \mathrm{i}(S(i) \neq 0) \cap \forall i(R(i)=0) \quad 1 \leq i \leq N$

All the proxy servers and mobile nodes in the cells of access points have no video clips to be played in switching zone. In this case, the AP with maximum $\mathbf{R}(\mathbf{i})$ is selected to get video clips from streaming media servers through proxy servers of maximum $\mathbf{P}(\mathbf{i})$ cell. In other words, the video clips to be played in switching zone should be downloaded to proxy servers by streaming media servers.

(3) $P(i) \geq P(j) \cap R(i)=1 \quad 1 \leq j \leq N$

Find the AP with maximum $\mathbf{P}(\mathbf{i})$, and the maximum $\mathbf{P}(\mathbf{i})$ cell contains the video clips that the mobile need play in the switching zone, and no data scheduling occurs between proxy servers. The detailed data scheduling mechanism refers to section 3.2.1 in this paper.

(4)P(i) $\geq P(j) \cap R(i)=0 \cap \exists j(R(j)=1) 1 \leq j \leq N$

Find the AP with maximum $\mathbf{P}(\mathbf{i})$, and the maximum $\mathbf{P}(\mathbf{i})$ cell does not contain the video clips that the mobile need play in the switching zone, but other proxy servers have these video clips. In this case, data scheduling occurs between proxy servers.

\section{SCHEDULING MECHANISM OF PROXY SERVERS AND ANALYSIS}

Two types of nodes, namely the proxy servers and mobile streaming media nodes, can provide video transmission service for mobile streaming media node in the system model. The data scheduling of proxy servers is dealt with in this section.

\subsection{Determination of Data Scheduling Size}

The mobile node Q3 moves towards lower right at an even speed of $\mathrm{V}$ from point $\mathrm{O}$ in cell 1, as shown in Fig. 4, and the direction

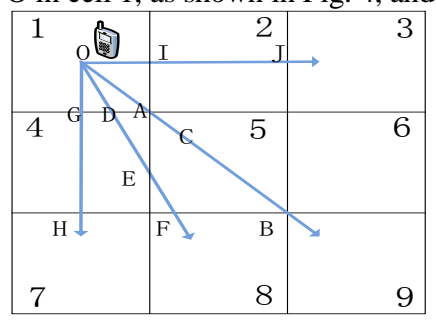

Fig. 4 Determination of data scheduling size

denotes the $90^{\circ}$ scope between $\mathrm{OJ}$ and $\mathrm{OH}$. If mobile node moves to point $\mathrm{B}$ along $\mathrm{OAB}$, it will only cross cell 1 and cell 5 . Line $\mathrm{AB}$ is the diagonal line and path length of square cell 5 . If the mobile node Q2 moves to point $\mathrm{F}$ along ODEF, it will cross the cell 1, cell 4 and cell 5 . The moving path in cell 1 is OD, in cell $4 \mathrm{DE}$, and in cell $5 \mathrm{EF}$

When mobile node enters cell 5 from any direction, the data scheduling will be conducted from proxy servers if cell 5 has no video clips the mobile node needs at this moment. In this case, the video clips corresponding to the moving time in cell 5 should be buffered into the proxy server in cell 5 . The video scheduling size depends on the stay time of mobile node in cell 5 . The stay time can be determined by the path length as for the case in Fig. 4 .

(1) Assume the video clip to be played in new cell boundary is the $\mathrm{M}^{\text {th }}$ one of clip series.

(2) Estimate the moving time of mobile node in new cell, $\mathrm{T}=\frac{|S|}{V}$.

(3) The video clips to be scheduled by the proxy server are

$$
\left(M+1, M+1+\frac{T}{t_{1}}\right)
$$

\subsection{Data Scheduling Mechanism and Analysis}

Definition 16:S1[i] denotes the video clip set scheduled for current cell.

Definition 17:AW $(I)$ denotes usable upload bandwidth size of proxy server of the $\mathrm{i}^{\text {th }}$ cell.

Definition 18: video clip proxy server ratio: the ratio of the number of proxy server with video clips to the number of total proxy servers in a cell.

The data scheduling size determined by section 4.1 is just a video clip set. As shown in Fig. 4, when the mobile node moves to point $\mathrm{B}$ along $\mathrm{OAB}$, it switches from cell 1 to cell 5 . If the proxy servers and mobile nodes in cell 5 have the needed video clip set, 
the $\operatorname{set}\left(M+1, M+1+\frac{T}{t_{1}}\right)$ should be scheduled from other proxy servers. The schematic procedures of data scheduling algorithm are described as follows:

(1) Sort the current video clip set $S 1[i]$ in ascending order in terms of video clip proxy server ratio of each clip.

(2) For $\mathrm{i}=1$ to $\mathrm{n}$

(3) Select the $i^{\text {th }}$ clip of $S 1[i]$ for data scheduling, and sieves out the usable proxy server set $\operatorname{SP} S_{i}[j]$.

(4) Sort SPS $S_{i}[j]$ in descending order in terms of usable upload bandwidth.

(5) Select the first proxy server of $\operatorname{SP}_{i}[j]$ to transmit the $i^{\text {th }}$ video clip.

(6) End for

The data scheduling mechanism by proxy server has the following advantages. Firstly, it improves the load balance of proxy servers because it selects the proxy server with maximum bandwidth capacity for data scheduling, which can basically make the bandwidth usage of proxy servers fair and the data transmission connections of proxy servers tend to be identical with each other. Secondly, it boosts the service ability because it gives priority to selecting the video clip with minimum video clip proxy server ratio, which enriches the scarce video clips in the streaming media network. When new mobile node needs such clips, more cells have them in proxy servers, which improve the service ability.

\section{TEST AND ANALYSIS}

\subsection{Test Design}

The test is conducted under the hardware of CPU IS Intel(R) Core(TM)2 Duo CPU T5750, 2.00GHZ, 2.0GB memory, with operation system of Windows XP Professional SP3. The model simulation is realized by JAVA in the platform of Eclipse. Several groups of comparison tests for AP selection mechanism are designed. The new AP selection mechanism is separately compared with the one only by signal intensity, the one only by video resource number and the one only by bandwidth size.

Assume $\mathrm{N}$ mobile nodes cross $\mathrm{M}$ AP-overlapped zones, the usable bandwidth capacity for $\mathrm{M}$ access points are $\mathbf{B W}[\boldsymbol{i}]$, and each mobile node needs bandwidth of bw for streaming media service. The signal intensity of each AP detected by each mobile node is denoted at random by $2 \mathrm{D}$ array of $\mathrm{S}[\mathrm{N}][\mathrm{M}]$, with $\mathrm{N}=30$, $\mathrm{M}=3$, and $\mathrm{bw}=2$ in this test. Let $\mathrm{BW}[0]=20, \mathrm{BW}[1]=40$, and $\mathrm{BW}[2]=70$ to represent the bandwidth difference of various cells. The signal intensity $\mathrm{S}[\mathrm{N}][\mathrm{M}]$ for $\mathrm{M}$ access points and $\mathrm{N}$ mobile nodes is generated at random, with $\mathrm{S}[0][\mathrm{M}]=$ random.nextInt(100), $\mathrm{S}[1][\mathrm{M}]=$ random.nextInt $(60), \quad \mathrm{S}[2][\mathrm{M}]=$ random.nextInt(30). The numbers of user video resources of various cells are $\mathrm{R}[0]=$ random.nextInt(30), $\quad \mathrm{R}[1]=$ random.nextInt(30), and $\mathrm{R}[2]=$ random.nextInt(30).

The test assumes the service congestion of mobile node as follows:

(1) $\mathrm{BW}[\mathrm{i}]=0$ denotes that the cell bandwidth resources are exhausted and cannot ensure the bandwidth service for new mobile node.

(2) $\mathrm{S}[\mathrm{N}][\mathrm{M}]<15$ denotes the mobile media and access points have weak signal, and the service will be congested if there are no streaming media.

(3) If $\mathrm{R}[\mathrm{i}]<10$ and no video resources requested in the cell are provided, the streaming media service will be congested.

\subsection{Result Analysis}

After the simulation program is finished, the number of mobile nodes connected to each cell and the number of mobile nodes congested in each cell are statistically analyzed in consideration of new AP selection mechanism, AP selection mechanism only by signal intensity, AP selection mechanism only by video resource number and AP selection mechanism only by bandwidth size. The statistical results are shown in Fig. 5, Fig. 6, Fig. 7, and Fig. 8.

The numbers of connected nodes for various mechanisms are shown in Fig. 5, Fig. 6, Fig. 7, and Fig. 8, with 11.7 nodes for signal intensity mechanism, 7.8 nodes for bandwidth mechanism, 8.9 nodes for video resource mechanism, and 17.2 nodes for new mechanism presented in this paper, which shows that the new mechanism reduces the congestion rate compared with other three mechanisms.

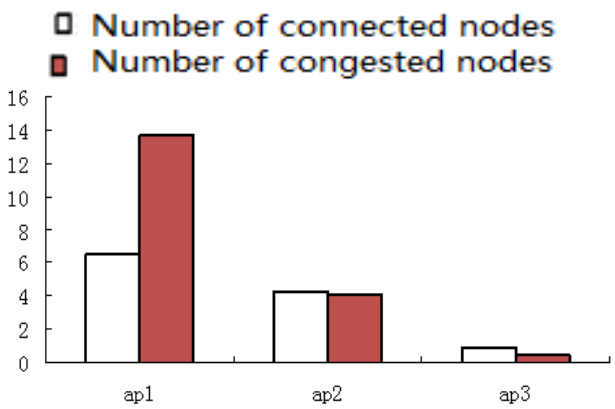

Fig. 5 Selection mechanism for signal intensity

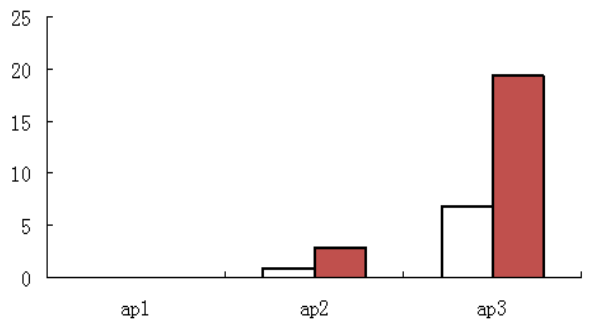

Fig. 6 Selection mechanism for bandwidth

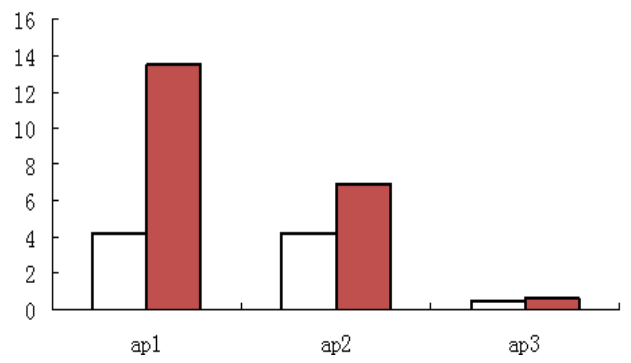

Fig. 7 Selection mechanism for clip number

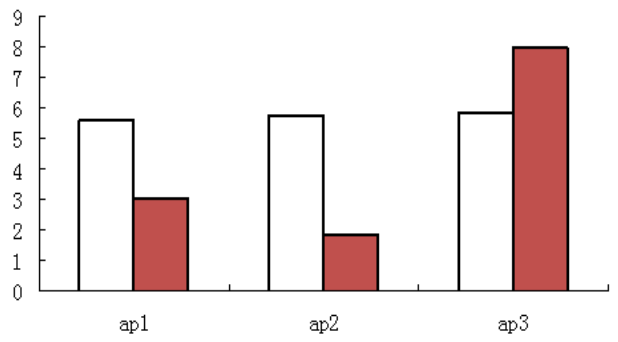

Fig. 8 Selection mechanism for new AP

As shown in Fig. 5, Fig. 6, Fig. 7, and Fig. 8, the new mechanism connects to nodes more evenly in ap1, ap2 and ap3 cells than other mechanisms do. The connected nodes by bandwidth mechanism are mostly at ap3 cell with more bandwidth, 
which causes heavy load on ap3 cell. However, the connected nodes by signal intensity mechanism and video resource mechanism are few in ap3 cell, which idles and wastes the bandwidth resources of ap3 cell. The analysis shows that the new mechanism can get better load balance than other three mechanisms and can improve the utilization of bandwidth to some extent.

\section{Conclusions}

The rapid development of mobile streaming media business and the increasing market demand bring much challenge against the application of mobile streaming media. In consideration of the environmental feature of mobile streaming media, this paper designs a new system model which is suitable for mobile streaming media and carries out the study on data scheduling of mobile streaming media. The data scheduling mechanisms for mobile nodes within cell and in the switching zone between cells are presented, which ensures the continuous playing of mobile node and improves the service quality of mobile streaming media to some extent. However, the research in how to ensure the service quality of mobile peer-to-peer streaming media is still in the preliminary stage, and there will be much work to be done in the future.

\section{REFERENCES}

[1] A.B. Smith, C.D. Jones, and E.F. Roberts, "Article Title," Journal, Publisher, Location, pp. 1-10, Date.

[2] Jones, C.D., A.B. Smith, and E.F. Roberts, Book Title, Publisher, Location, Date.

[3] Li Zhuo, Jin Wang, David Dagan Feng, et al. "Wireless Media Streaming System over CDMA Networks", Proceedings of Advanced Communication Technology, the 9th International Conference on Gang Won-Do, pp. 2226-2230, 2007.

[4] Miikka Lundan, logor D.D.Curcio. "Mobile Streaming Services in WCDMA Networks", Proceedings of 10th IEEE Symposium on Computers and Communications, pp. 231-236, 2005.
[5] Palau C, Martinez-Nohales, Mares, et al. "On Mobile Video Streaming IPTV", Proceedings of 10th International Conference of Telecommunications ConTEL on Zagreb, pp. 457-462, 2009.

[6] Canali C,Renda M E,Santi P,et al. "Enabling efficient peer-topeer resource sharing in wireless mesh networks", Mobile Computing, IEEE Transactions, pp. 333-347, 2010,9(3).

[7] Zhu Y,Zeng W, Liu H, et al. "Supporting video streaming services in infrastructure wireless mesh networks: architecture and protocols", Communications, ICC.08, IEEE International Conference, pp. 1850-1855, 2008.

[8] Zhao J, Zhang P, Cao G. "On cooperative caching in wireless P2P networks", Distributed Computing Systems, 2008. ICDCS'08, The 28th International Conference on IEEE, pp. 731-739, 2008.

[9] Andersen F U, de Meer H, Dubinsky I,et al. "An Architecture Concept for Mobile P2P File Sharing Services", GI Jahrestagung (2), pp. 229-233, 2004,51.

[10] Peer-to-Peer[EB/OL], http://en.wikipedia.org/wiki/Peer-topeer, 2014-02-26.

[11] Tarik Taleb, Kazuo Hashimoto. "A New Real-Time MultiSource Mobile-Streaming Architecture", IEEE TRANSACTIONS ON BROADCASTING, pp. 662-673, VOL. 57, NO. 3, 2011.

[12] HUANG Xiao-tao, ZHANG Wei, ZHU Hua, LU Zheng-ding. "Layered Multi-description Coding Based on P2P Video Streaming", Computer Science, pp. 65-69, 2009, 36(12).

[13] HUANG Xiao-tao, CHENG Xiao-chen, LU Zheng-ding. "Layered and Multiple Description Coding Based Dynamic Bandwidth Allocation strategy", Journal of Chinese computer systems, pp. 1030-1034, 2010, Vol.3, No.6.

[14] Yan Zhang. "Handoff Performance in Wireless Mobile Networks with Unreliable Fading Channel", IEEE Transactions on Mobile Computing, pp. 188-210, 2010, 9(2). 